\title{
Never Too Soon: An Unusual Case of Intrahepatic Cholestasis of Pregnancy at Five Weeks Gestation
}

\author{
Nicha Wongjarupong ${ }^{1}$, Sheila Bharmal ${ }^{2}$, Nicholas Lim ${ }^{2}$ \\ 1. Internal Medicine, University of Minnesota, Minneapolis, USA 2. Division of Gastroenterology, Hepatology and \\ Nutrition, University of Minnesota, Minneapolis, USA
}

Corresponding author: Nicha Wongjarupong, nwongjarupong@gmail.com

\begin{abstract}
Intrahepatic cholestasis of pregnancy (ICP) is an incompletely understood liver disease which results in systemic accumulation of bile acids, associated with maternal pruritus and increased risk of intrauterine fetal death. Onset is typically in the third trimester; however, rare cases have been reported early in the first trimester. We present a case of severe, early onset ICP at five weeks gestation in a spontaneous pregnancy. The patient was treated successfully with ursodeoxycholic acid and, after close obstetrical surveillance, delivered a healthy female infant via induced delivery at 34 weeks six days.
\end{abstract}

Categories: Obstetrics/Gynecology, Gastroenterology

Keywords: obstetric cholestasis, liver disease of pregnancy

\section{Introduction}

Intrahepatic cholestasis of pregnancy (ICP) is a liver disease of undefined etiology and pathogenesis, typically associated with maternal pruritus involving the palms and soles, combined with elevated serum bile acid levels of $\geqslant 10 \mu \mathrm{mol} / \mathrm{L}$.

In general, the maternal course of ICP is benign, with resolution of clinical symptoms and biochemical abnormalities after delivery. Conversely, ICP has more serious consequences for the fetus including increased risk of spontaneous pre-term delivery, lower birth weight, cardiac arrhythmias, meconiumstaining of amniotic fluid, infant respiratory distress syndrome, and sudden perinatal death [1-3]. Severe ICP is defined as serum bile acids of more than $40 \mu \mathrm{mol} / \mathrm{L}$. Severe cholestasis is seen in approximately one-fifth of all cases of ICP and is associated with worse fetal outcomes $[1,4,5]$.

ICP typically occurs during the late second to third trimester of pregnancy. Genetic defects in bile canaliculi transporters such as ATP8B1, ABCB11, ABCB4, ABCC2, NR1H4, and FGF19 can be found in patients with ICP [6]. Estrogen is postulated to play an important role in the onset of ICP given the association of ICP with multi-parity, ovarian hyper-stimulation and prior use of oral contraceptives [7]. ICP typically presents during late pregnancy as estrogen levels start to rise at six to eight weeks gestation and peak at 36 weeks [8]. In the literature review, we found a total of 10 cases of severe ICP during the first trimester reported previously in the literature, of which, our case report at five weeks gestation is the earliest onset of ICP thus far [8-14].

Received 09/02/2020 Review began 09/08/2020
Review ended 09/17/2020 Published 09/19/2020

\section{() Copyright 2020}

Wongjarupong et al. This is an open access article distributed under the terms of the Creative Commons Attribution License CC-BY 4.0., which permits unrestricted use, distribution, and reproduction in any medium, provided the original author and source are credited.
We present a case of severe ICP with symptom onset as early as five weeks gestation prior to obstetric confirmation of pregnancy. Our aim is to raise awareness of this rare presentation of ICP during the early pregnancy, as it is a treatable condition with a favorable outcome if detected.

\section{Case Presentation}

A 32-year-old, otherwise healthy gravida 2 para 1, white female at approximately five weeks gestation presented to a local emergency department for pruritus predominantly involving her extremities. At the time, the patient and treating physician were not aware of her pregnancy status. Physical exam was unremarkable and no further testing was pursued. The patient was discharged with a presumed allergic reaction. She completed a short course of prednisone and diphenhydramine without improvement, prompting further visits to the emergency department for similar symptoms.

Approximately four weeks after initial presentation, the patient developed nausea and epigastric pain. Urine pregnancy testing was positive. Obstetrical ultrasound confirmed a nine-week gestation monofetal pregnancy. Laboratory testing revealed a total bilirubin $6.1 \mathrm{mg} / \mathrm{dL}(0.2-1.2 \mathrm{mg} / \mathrm{dL})$, direct bilirubin $4.5 \mathrm{mg} / \mathrm{dL}$ (0.1-0.5), indirect bilirubin $1.6 \mathrm{mg} / \mathrm{dL}(0.2-0.8)$, alkaline phosphatase $179 \mathrm{IU} / \mathrm{L}$ (50-136 IU/L), aspartate aminotransferase 1117 IU/L (2-40 IU/L), alanine aminotransferase (ALT) $1406 \mathrm{IU} / \mathrm{L}$ (8-45 IU/L) and international normalized ratio 1 (0.86-1.14). Abdominal ultrasound showed mild diffuse gallbladder wall thickening without cholelithiasis. The common bile duct was normal in caliber and Doppler evaluation showed patent vasculature. Subsequent magnetic resonance cholangiopancreatography was also normal. 


\section{Cureus}

The patient denied alcohol, acetaminophen or any other toxin use. Extensive laboratory evaluation including viral hepatitis testing (A, B, C, E, Epstein-Barr virus, cytomegalovirus, herpes simplex virus) and autoimmune serologies (ANA, anti-smooth muscle antibody, F-actin antibody IgG, serum immunoglobulin levels, anticardiolipin, beta-2 glycoprotein, SS-A antibody, SS-B antibody, Jo-1 antibody) were negative. Serum ceruloplasmin and alpha-1-antitrypsin were normal. Total bile acids later returned at $462 \mu \mathrm{mol} / \mathrm{L}$ supporting a diagnosis of severe ICP. Liver biopsy was pursued given the patient's atypical presentation. Histopathology showed mild to moderately severe non-specific lobular hepatitis without evidence of chronic liver disease (Figures 1,2).

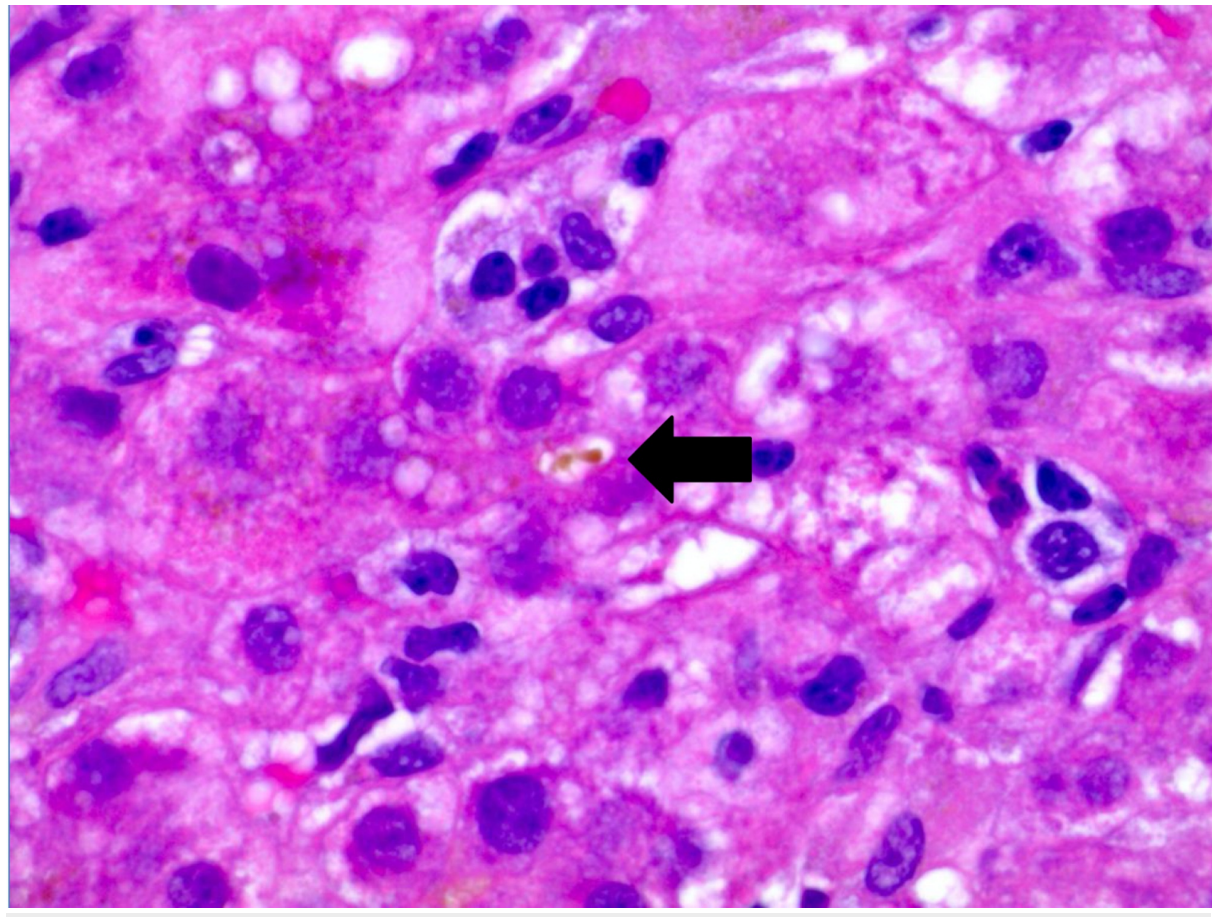

FIGURE 1: Liver biopsy with canalicular cholestasis (at arrow), H\&E, $1000 x$

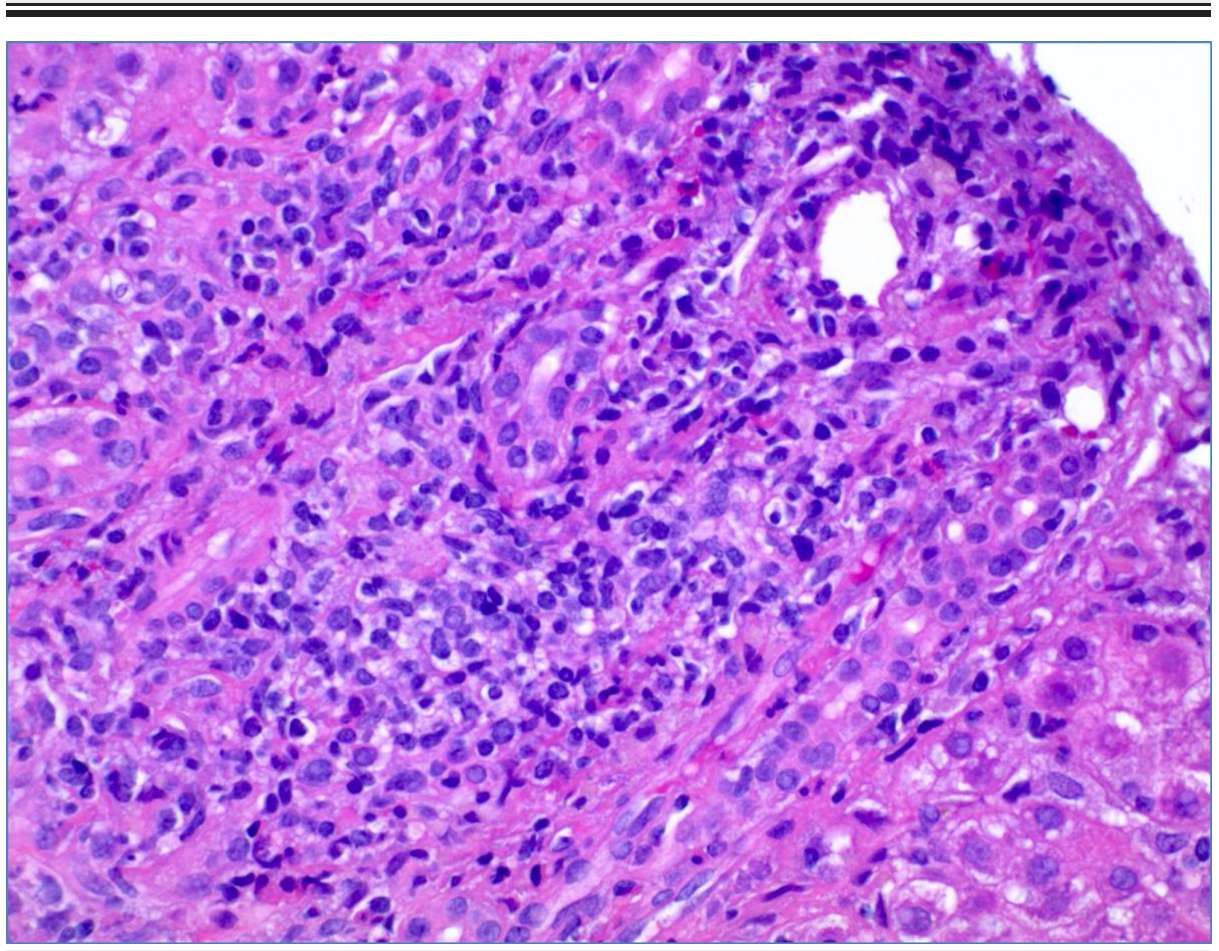

FIGURE 2: Liver biopsy with mononuclear inflammation and intact bile duct, H\&E, 400x 
The patient was treated for ICP with ursodeoxycholic acid (UDCA) at $600 \mathrm{mg}$ twice daily with gradual improvement in her pruritus and normalization of liver function tests late in her second trimester. Total bile acid level was monitored periodically and also progressively improved until the time of delivery.

After close obstetrical and biochemical surveillance of the maternal and fetal conditions, the patient was induced at 34 weeks 6 days gestation. Dexamethasone was administered to accelerate fetal lung maturation. The patient delivered a healthy female infant weighing 5 pounds 15 ounces via uncomplicated vaginal delivery. Both patient and baby followed a routine post-partum course with full resolution of symptoms and liver biochemical abnormalities.

\section{Discussion}

ICP is the most common pregnancy-associated liver disease, with a variable incidence worldwide between $0.2 \%$ and $25 \%$ [15]. The pathophysiology of ICP is incompletely understood. Family clustering and varying incidence in different geographic regions suggest an underlying genetic predisposition. Moreover, genetic defects in at least six canalicular transporters have been identified as associated with ICP [6]. Our patient did not have any obvious risk factors for ICP, and genetic testing was not pursued given that this was her first presentation with ICP.

A diagnosis of ICP should be considered in pregnant patients with intense pruritus of the extremities, concentrated primarily on the hands and soles, in the absence of rash. As the disease progresses, secondary skin changes can range from excoriation to prurigo nodules [16]. Serum bile acid level exceeding $10 \mathrm{~mol} / \mathrm{L}$ is the gold standard for diagnosis. Higher bile acid levels $(>40 \mu \mathrm{mol} / \mathrm{L})$ are associated with increased rates of spontaneous preterm delivery and asphyxia events $[1,4,5]$. Biochemical abnormalities are variable and mostly resolve completely after delivery. Serum aminotransferases are usually less than two times the upper limit of normal but may reach values greater than $1000 \mathrm{IU} / \mathrm{L}$. Bilirubin is increased only in exceptional cases $[6]$.

ICP rarely occurs during the first trimester. Our patient first developed pruritus at five weeks gestation and is the earliest reported case of ICP from a spontaneous pregnancy [8]. The onset of ICP in the second and third trimesters is hypothesized to be related to the peak estrogen levels that occur during the later stages of pregnancy $[7,8]$. Patients who present in the first trimester potentially could have other factors causing elevated estrogen. Our patient did not have any of the defined risk factors for hyperestrogenemia. Of the previous reported cases of first trimester severe ICP, four out of 10 patients underwent ovarian stimulation for in vitro fertilization (IVF) and one patient had a twin pregnancy [8,13]. In women undergoing IVF, additional follicle-stimulating hormone induces an estrogen surge to stimulate ovulation [17]. Only one patient underwent genetic testing which showed homozygous MDR3, and homozygous BSEP mutation, both of which were previously identified in the ICP cohort [10].

On review of 10 cases with first trimester severe ICP, gestational age ranged from five to 11 weeks and maternal age ranged from 21 to 32 years [8-14]. Regarding outcomes, there were no cases of maternal death. There were five patients who had term deliveries, two patients who had preterm delivery, one patient underwent pregnancy termination at 11 weeks due to persistent elevated liver enzymes despite UDCA treatment, one patient who had a blighted ovum, and one patient who developed a spontaneous miscarriage.

Management of ICP is focused on close obstetrical, clinical and biochemical surveillance. Treatment with UDCA improves both transport and secretion of bile acids, minimizing fetal exposure. UDCA is effective in reducing pruritus and improving liver biochemical abnormalities in patients with ICP. It also lowers the rate of prematurity and decreases the need for neonatal intensive care unit admission [18]. Moreover, UDCA is safe and well-tolerated [19]. Earlier delivery is indicated in patients with intractable pruritus, persistence of hepatic dysfunction and past history of bad neonatal outcome due to ICP. The ideal gestational age for elective induction of labor to minimize the risk of perinatal mortality is unknown, but a large cohort study has suggested that delivery at 36 weeks gestation may be optimal [20].

\section{Conclusions}

In conclusion, we present an unusual case of severe, early onset ICP at five weeks gestation prior to obstetric confirmation of pregnancy. ICP remains an enigmatic disease with variable clinical presentations and potentially serious fetal outcomes. Other types of liver disease should always be excluded and treatment with UDCA results in resolution of the symptoms and elevated liver enzymes in the majority of cases. In any female patient of childbearing age presenting with generalized pruritus, a diagnosis of ICP should always be considered regardless of known pregnancy status or the trimester of pregnancy.

\section{Additional Information \\ Disclosures}


Human subjects: Consent was obtained by all participants in this study. N/A issued approval N/A. Informed consent was obtained verbally from the patient for case publication. The consent was obtained during the patient encounter. However, with multiple attempts, we could not contact the patient to later obtain the signed inform consent. There is no patient's identifying information included in the article. Conflicts of interest: In compliance with the ICMJE uniform disclosure form, all authors declare the following: Payment/services info: All authors have declared that no financial support was received from any organization for the submitted work. Financial relationships: All authors have declared that they have no financial relationships at present or within the previous three years with any organizations that might have an interest in the submitted work. Other relationships: All authors have declared that there are no other relationships or activities that could appear to have influenced the submitted work.

\section{Acknowledgements}

We would like to thank Schuyler O Sanderson, MD of Hospital Pathology Associates in Minneapolis, MN for kindly providing the pathology images.

\section{References}

1. Brouwers L, Koster MP, Page-Christiaens GC, et al.: Intrahepatic cholestasis of pregnancy: maternal and fetal outcomes associated with elevated bile acid levels. Am J Obstet Gynecol. 2015, 212:100.E1-100.E7. 10.1016/j.ajog.2014.07.026

2. Fisk NM, Bye WB, Storey GN: Maternal features of obstetric cholestasis: 20 years experience at King George V Hospital. Aust N Z J Obstet Gynaecol. 1988, 28:172-176. 10.1111/j.1479-828x.1988.tb01657.x

3. Lin J, Gu W, Hou Y: Diagnosis and prognosis of early-onset intrahepatic cholestasis of pregnancy: a prospective study. J Matern Fetal Neonatal Med. 2019, 32:997-1003. 10.1080/14767058.2017.1397124

4. Walker IA, Nelson-Piercy C, Williamson C: Role of bile acid measurement in pregnancy . Ann Clin Biochem. 2002, 39:105-113. 10.1258/0004563021901856

5. Glantz A, Marschall HU, Mattsson LA: Intrahepatic cholestasis of pregnancy: relationships between bile acid levels and fetal complication rates. Hepatology. 2004, 40:467-474. 10.1002/hep.20336

6. Floreani A, Gervasi MT: New insights on intrahepatic cholestasis of pregnancy . Clin Liver Dis. 2016, 20:177189. 10.1016/j.cld.2015.08.010

7. Salat-Baroux J, Cornet D, Alvarez S, Antoine JM, Mandelbaum J, Plachot M: Hormonal secretions in singleton pregnancies arising from the implantation of fresh or frozen embryos after oocyte donation in women with ovarian failure. Fertil Steril. 1992, 57:150-155. 10.1016/s0015-0282(16)54792-x

8. Mutlu MF, Aslan K, Guler I, Mutlu I, Erdem M, Bozkurt N, Erdem A: Two cases of first onset intrahepatic cholestasis of pregnancy associated with moderate ovarian hyperstimulation syndrome after IVF treatment and review of the literature. J Obstet Gynaecol. 2017, 37:547-549. 10.1080/01443615.2017.1286302

9. Hubschmann AG, Orzechowski KM, Berghella V: Severe first trimester recurrent intrahepatic cholestasis of pregnancy: a case report and literature review. AJP Rep. 2016, 6:38-41. 10.1055/s-0035-1565922

10. Keitel V, Vogt C, Häussinger D, Kubitz R: Combined mutations of canalicular transporter proteins cause severe intrahepatic cholestasis of pregnancy. Gastroenterology. 2006, 131:624-629. 10.1053/j.gastro.2006.05.003

11. Stulic M, Culafic D, Boricic I, et al.: Intrahepatic cholestasis of pregnancy: a case study of the rare onset in the first trimester. Medicina. 2019, 55:454. 10.3390/medicina55080454

12. Johnston RC, Stephenson ML, Nageotte MP: Novel heterozygous ABCB4 gene mutation causing recurrent first-trimester intrahepatic cholestasis of pregnancy. J Perinatol. 2014, 34:711-712. 10.1038/jp.2014.86

13. Zamah AM, El-Sayed YY, Milki AA: Two cases of cholestasis in the first trimester of pregnancy after ovarian hyperstimulation. Fertil Steril. 2008, 90:1202.E7-1202.E10. 10.1016/j.fertnstert.2007.08.072

14. Brites D, Rodrigues CM, Cardoso M da C, Graça LM: Unusual case of severe cholestasis of pregnancy with early onset, improved by ursodeoxycholic acid administration. Eur J Obstet Gynecol Reprod Biol. 1998, 76:165-168. 10.1016/s0301-2115(97)00185-1

15. Geenes V, Williamson C: Intrahepatic cholestasis of pregnancy. World J Gastroenterol. 2009, 15:2049-2066. 10.3748/wig.15.2049

16. Chao TT, Sheffield JS: Primary dermatologic findings with early-onset intrahepatic cholestasis of pregnancy . Obstet Gynecol. 2011, 117:456-458. 10.1097/AOG.0b013e3181f61b11

17. Henriksson P: Cardiovascular problems associated with IVF therapy (PREPRINT) . J Intern Med. 2020, 10.1111/joim.13136

18. Grand'Maison S, Durand M, Mahone M: The effects of ursodeoxycholic acid treatment for intrahepatic cholestasis of pregnancy on maternal and fetal outcomes: a meta-analysis including non-randomized studies. J Obstet Gynaecol Can. 2014, 36:632-641. 10.1016/s1701-2163(15)30544-2

19. Bacq Y, Sentilhes L, Reyes HB, et al.: Efficacy of ursodeoxycholic acid in treating intrahepatic cholestasis of pregnancy: a meta-analysis. Gastroenterology. 2012, 143:1492-1501. 10.1053/j.gastro.2012.08.004

20. Puljic A, Kim E, Page J, Esakoff T, Shaffer B, LaCoursiere DY, Caughey AB: The risk of infant and fetal death by each additional week of expectant management in intrahepatic cholestasis of pregnancy by gestational age. Am J Obstet Gynecol. 2015, 212:667.E1-667.E5. 10.1016/j.ajog.2015.02.012 